\title{
Dramático engaño / El paso y peso de los años
}

María Daniela Alpizar Hidalgo

Costa Rica

maria.alpizar.hidalgo@est.una.ac.cr

Resumen: El siguiente poema fue escrito en el 2012, durante una clase de matemáticas. Cuando observaba que una pareja de amigos se ignoraba mutuamente, tras una pelea, debido a una situación de celos. Cada uno me compartió su versión por separado, lo que causó que comprendiera más su situación sentimental. Claramente, la poesía nunca deja el propio prejuicio de lado, así que este también es su posición.

Como mujeres y hombres adultos, quienes comienzan una eterna preocupación de lo arrugado de sus caras, sus patas de gallo cada día más pronunciadas, sus manos menos jóvenes. ¿Se me nota las patas de gallo? ¡Esta crema no me esta funcionando, cada día más arrugas! ¡que ojeras, cada vez se me notan más! ¡Parezco de 70, con este arrugero! ¡Sí se ríe mucho, se arruga!

Tras comentarios como estos y muchos más, nace la interrogante del surgimiento de las arrugas y cómo determinan vergüenza al identificarles. En el intercambio con mi abuela, compartimos que las arrugas o líneas de expresión no son muestra de fealdad o descuido, sino de la naturalidad y el significado que estas portan: premio, triunfo que no se hace de un día a otro. Por este significado, nace en el 2009, el siguiente poema:

Abstract: The following poem was written in 2012, during a math class, when I noticed that two friends were ignoring each other after an argument, due to a jealousy issue. 
Each one separately told me his own version, which allowed me to have a better understanding of their sentimental situation. Clearly, poetry never leaves aside your own perspective, so it also reflects my position.

As adult women and men who start their never-ending concern about wrinkles on their faces, their crow's feet, which become more and more noticeable every day, and their aging hands. Can you tell I have crow's feet? This lotion is not working; I have more wrinkles every day! Look at those shadows under my eyes! They are getting darker and darker! I look 70, I have so many wrinkles! If you laugh a lot, you will get wrinkles!

After comments like these and many more, the question arises regarding the appearance and how they determine shame when identifying them. In a conversation with my grandmother, we agreed that wrinkles or expression lines are not signs of ugliness or neglect, but of naturalness and of the meaning that they carry: rewards, success that is not achieved overnight. This poem arises from this meaning, in 2009:

\section{Dramático engaño}

Estás con ella

Yo con él.

Sin felicidad

Sin diversión

Abusamos de la libertad

para entregar deseos vacíos,

caprichos inalienables.

Yo aquí

Tú allá

Cerca pero tan lejos,

a unos metros, pero en realidad kilómetros

rosándonos con miradas en este campo de batalla,

cubiertos por el mismo techo que no sé de qué

nos protege,

porque ya siento una tempestad.

Besándola, yo

Besándolo

Besos sucios sin razón

Sin sabor

Sin corazón.

Escondiendo sentimientos auténticos. 
Fría pasión que no se siente

yo aquí, tú allá

en este pecado

en esta tentación que quema el alma y derrite el corazón

en la más vil de su interacción,

y provoca una ira compulsiva

que es una verdadera guerra que mata a su paso.

Quemándonos bajo el mismo pecado.

Tú con ella

Yo con él.

\section{El paso y peso de los años}

¿Líneas de expresión o arrugas?

Da lo mismo, son lo mismo

Pequeñas rectas en la piel

que expresan los años de algunos tiempos.

Reflejando en su exposición

lo que vivimos y aprendemos

de cada buen y mal paso.

Una línea de expresión hay en mi cara

o mi cara tiene líneas de reflexión,

que más da, son lo mismo,

expresan un pasado que ya se disfrutó.

En mi piel parto el recuerdo de mis 50,

de mis 60 ,

de mis mil experiencias y anécdotas

como equipaje vanidoso que no se desprende.

¿Una fotografía?

la desconocía,

por eso cuando quiero recordar

veo mi piel manchada

llena de arrugas y cicatrices que hoy con honor poseo.

Sé que mi espíritu tiene una sabiduría como ningún otra, que solo se demuestra en las líneas de expresión o arrugas,

da lo mismo, son lo mismo.

Orgullosa estoy, mis años los colecciono como pequeñas rectas en mi piel.

¿Líneas de expresión o arrugas?

Da lo mismo, son lo mismo. 